\title{
An Example of a Quasigroup with a Distributive Subquasigroup Lattice
}

\author{
Konrad Pióro
}

\begin{abstract}
We first show (using ideas from Pióro, in Quasigroups Relat Syst 15:309-316, 2007) that if a finitely generated quasigroup or more general, an algebra satisfying some quasigroup-like condition, $A$ has a distributive subalgebra lattice and satisfies the descending chain condition for finitely generated subalgebras, then $A$ is cyclic (i.e. has one generator). But the main aim of the paper is to construct a two-generated non-cyclic quasigroup with a distributive subquasigroup lattice (as a part of this construction we will obtain an analogous example for groupoids). Thus distributivity of subquasigroup lattice is an essentially weaker condition than in the case of groups (it is well-know that such groups are locally cyclic).
\end{abstract}

Mathematics Subject Classification (2000). Primary 08A30, 20N05; Secondary 05B15, 06D05, 06B15.

Keywords. Quasigroup, cyclic quasigroup, subquasigroup lattice, subalgebra lattice, distributive lattice.

\section{Introduction}

It is a classical result due to Ore (see e.g. [4]) that the subgroup lattice of a group $\mathcal{G}$ is distributive if and only if $\mathcal{G}$ is locally cyclic (i.e. each finitely generated subgroup of $\mathcal{G}$ is cyclic). In [3] we have shown that if a finite quasigroup $\mathcal{Q}$ has a distributive subquasigroup lattice, then $\mathcal{Q}$ is cyclic (and also each of its subquasigroups is cyclic). On the other hand, we have constructed in [3] a four-element (and five-element commutative) quasigroup with a non-distributive subquasigroup lattice, such that each of its subquasigroups is cyclic.

Now, using ideas from [3], we first generalize this result for finitely generated quasigroups satisfying the descending chain condition for finitely generated subquasigroups. In fact, we generalize this result for finitely generated 
algebras of arbitrary types which additionally satisfy some quasigroup-like condition.

But the main aim of the paper is to construct a two-generated non-cyclic quasigroup with a distributive subquasigroup lattice (as a part of this construction we will obtain an analogous example for groupoids). Thus the class of quasigroups with distributive subquasigroup lattices is more complicated and interesting than the class of groups with distributive subgroup lattices.

An algebra A satisfies the descending chain condition for (finitely generated) subalgebras (see e.g. [2]) if there does not exist a strictly descending sequence of (finitely generated) subalgebras in $\mathbf{A}$.

For elements $a_{1}, \ldots, a_{k}$ of an algebra $\mathbf{A}$, by $\left\langle a_{1}, \ldots, a_{k}\right\rangle$ (or more formally by $\left.\left\langle a_{1}, \ldots, a_{k}\right\rangle_{\mathbf{A}}\right)$ we denote the subalgebra of $\mathbf{A}$ generated by $a_{1}, \ldots, a_{k}$.

Theorem 1.1. Let $\mathbf{A}=\left(A,\left(\varphi^{\mathbf{A}}\right)_{\varphi \in K}\right)$ be an algebra such that

(i) A is finitely generated,

(ii) A satisfies the descending chain condition for finitely generated subalgebras,

(iii) the subalgebra lattice of $\mathbf{A}$ is distributive,

(iv) for each $a, b \in A$, there exists $c \in\langle a, b\rangle$ such that $b \in\langle a, c\rangle$ and $a \in\langle b, c\rangle$. Then $\mathbf{A}$ is cyclic (i.e. has one generator).

Each finitely generated subalgebra of $\mathbf{A}$ also satisfies (i)-(iv), so it is cyclic, too. Next, (iv) is necessary, e.g. a two-element algebra without operations (or with projections only) satisfies (i)-(iii).

Proof. It is sufficient to show that each two-generated subalgebra of $\mathbf{A}$ is cyclic. Assume otherwise. Then by (ii), there is a minimal (up to inclusion) such subalgebra $\mathbf{B}$. Using again (ii) we can choose generators $u, w$ of $\mathbf{B}$ such that for each $u_{1}, w_{1} \in B$, if $\left\langle u_{1}, w_{1}\right\rangle=\mathbf{B}$ and $\left\langle u_{1}\right\rangle \leq\langle u\rangle$, then $\left\langle u_{1}\right\rangle=\langle u\rangle$.

By (iv) there is $v \in\langle u, w\rangle$ such that $\langle u, v\rangle=\langle w, v\rangle=\langle u, w\rangle=\mathbf{B}$. Since the subalgebra lattice of $\mathbf{A}$ is distributive, $\langle v\rangle=\langle v\rangle \wedge\langle u, w\rangle=\langle v\rangle \wedge$ $(\langle u\rangle \vee\langle w\rangle)=(\langle v\rangle \wedge\langle u\rangle) \vee(\langle v\rangle \wedge\langle w\rangle)$. Let $\mathbf{B}_{1}=\langle v\rangle \wedge\langle u\rangle=\langle v\rangle \cap\langle u\rangle$ and $\mathbf{B}_{2}=\langle v\rangle \wedge\langle w\rangle=\langle v\rangle \cap\langle w\rangle$. Of course $u \notin\langle v\rangle$, so $\mathbf{B}_{1} \supsetneqq\langle u\rangle$.

Since $\langle v\rangle=\mathbf{B}_{1} \vee \mathbf{B}_{2} \leq \mathbf{B}_{1} \vee\langle w\rangle=\left\langle B_{1} \cup\{w\}\right\rangle$, there are $b_{1}, \ldots, b_{j} \in B_{1}$ such that $v \in\left\langle w, b_{1}, \ldots, b_{j}\right\rangle$. Further, $\left\langle b_{1}, \ldots, b_{j}\right\rangle \leq \mathbf{B}_{1} \varsubsetneqq\langle u\rangle \leq \mathbf{B}$. Thus $\left\langle b_{1}, \ldots, b_{j}\right\rangle$ is cyclic, because otherwise it would contain a non-cyclic two-generated subalgebra which would be less then $\mathbf{B}$. Let $b$ be an element of $\mathbf{B}_{1}$ such that $\left\langle b_{1}, \ldots, b_{j}\right\rangle=\langle b\rangle$. Then $\mathbf{B}=\langle b, w\rangle$, but $\langle b\rangle \leq \mathbf{B}_{1} \supsetneqq\langle u\rangle$, a contradiction with the choice of $u$ and $w$.

Corollary 1.2. Let a finite algebra $\mathbf{A}$ satisfy (iii), (iv). Then $\mathbf{A}$ and each of its subalgebras are cyclic. Thus

Note that (iv) of Theorem 1.1 holds for each quasigroup (for $c=a \circ b$ ).

Corollary 1.3. Let a quasigroup $\mathcal{Q}$ satisfy (i), (ii), (iii). Then $\mathcal{Q}$ is cyclic. 
For quasigroups we can take $v=u \circ w$ in the proof of Theorem 1.1. Next, the distributive law is used only to obtain $\langle v\rangle=(\langle v\rangle \wedge\langle u\rangle) \vee(\langle v\rangle \wedge\langle w\rangle)$. Thus (iii) can be replaced by

(iii') for all elements $p, q,\langle p \circ q\rangle=(\langle p \circ q\rangle \wedge\langle p\rangle) \vee(\langle p \circ q\rangle \wedge\langle q\rangle)$.

The above result is proved in [3] for finite quasigroups. Moreover, a six-element commutative quasigroup is constructed in [3] which satisfies (iii'), but its subquasigroup lattice is isomorphic to the five-element non-distributive lattice $\mathcal{M}_{5}$.

The next result is formulated and proved in more general case of partial algebras, because it will be needed in next sections. Let $k^{\mathbf{A}}$ be a partial operation of a partial algebra $\mathbf{A}$. Then by $\operatorname{dom}\left(k^{\mathbf{A}}\right)$ we denote the domain of $k^{\mathbf{A}}$, i.e. the set of all $\left(a_{1}, \ldots, a_{\kappa(k)}\right) \in A^{\kappa(k)}$ such that $k^{\mathbf{A}}\left(a_{1}, \ldots, a_{\kappa(k)}\right)$ is defined (where $\kappa(k)$ is the arity of $k^{\mathbf{A}}$ ). Analogously, $\operatorname{dom}\left(t^{\mathbf{A}}\right)$ denotes the domain of a term $t$.

Theorem 1.4. A partial algebra $\mathbf{A}=\left(A,\left(k^{\mathbf{A}}\right)_{k \in K}\right)$ has a distributive subalgebra lattice if and only if for each operation $k^{\mathbf{A}},\left(a_{1}, \ldots, a_{\kappa(k)}\right) \in \operatorname{dom}\left(k^{\mathbf{A}}\right)$ and for each $b \in\left\langle k^{\mathbf{A}}\left(a_{1}, \ldots, a_{\kappa(k)}\right)\right\rangle, \quad b \in \bigvee_{i=1}^{\kappa(k)}\left(\langle b\rangle \wedge\left\langle a_{i}\right\rangle\right)$.

Proof. $\Longrightarrow$ follows immediately from distributive laws.

$\Longleftarrow$. Take subalgebras $\mathbf{B}, \mathbf{C}, \mathbf{D}$. We have to show $\mathbf{D} \wedge(\mathbf{B} \vee \mathbf{C}) \leq(\mathbf{D} \wedge \mathbf{B}) \vee$ $(\mathbf{D} \wedge \mathbf{C})$. Each element of $\mathbf{B} \vee \mathbf{C}=\langle B \cup C\rangle$ is a value of some term $t$ on some elements from $B \cup C$. Thus it is sufficient to prove that for each term $t$ and $a_{1}, \ldots, a_{m} \in B \cup C$ (where $m$ is the arity of $t$ ), if $\left(a_{1}, \ldots, a_{m}\right) \in \operatorname{dom}\left(t^{\mathbf{A}}\right)$, then $\mathbf{D} \wedge\left\langle t^{\mathbf{A}}\left(a_{1}, \ldots, a_{m}\right)\right\rangle \leq(\mathbf{D} \wedge \mathbf{B}) \vee(\mathbf{D} \wedge \mathbf{C})$. It is obvious if $t\left(x_{1}, \ldots, x_{m}\right)=x_{i}$ for some $1 \leq i \leq m$. Thus let $t\left(x_{1}, \ldots, x_{m}\right)=k\left(t_{1}\left(x_{1}, \ldots, x_{m}\right), \ldots, t_{n}\left(x_{1}, \ldots, x_{m}\right)\right)$ (where $n$ is the arity of $k$ ) and assume that our thesis is true for $t_{1}, \ldots, t_{n}$. If $\left(a_{1}, \ldots, a_{m}\right) \in \operatorname{dom}\left(t^{\mathbf{A}}\right)$, then $\left(a_{1}, \ldots, a_{m}\right) \in \operatorname{dom}\left(t_{i}^{\mathbf{A}}\right)$ for $i=1, \ldots, n$ and $\left(t_{1}^{\mathbf{A}}\left(a_{1}, \ldots, a_{m}\right), \ldots, t_{n}^{\mathbf{A}}\left(a_{1}, \ldots, a_{m}\right)\right) \in \operatorname{dom}\left(k^{\mathbf{A}}\right)$. Take $d \in \mathbf{D} \wedge\left\langle t^{\mathbf{A}}\left(a_{1}, \ldots\right.\right.$, $\left.\left.a_{k}\right)\right\rangle$. Then $d \in\left(\langle d\rangle \wedge\left\langle t_{1}^{\mathbf{A}}\left(a_{1}, \ldots, a_{m}\right)\right\rangle\right) \vee \ldots \vee\left(\langle d\rangle \wedge\left\langle t_{n}^{\mathbf{A}}\left(a_{1}, \ldots, a_{m}\right)\right\rangle\right)$. Next, by induction hypothesis, $\langle d\rangle \wedge\left\langle t_{i}^{\mathbf{A}}\left(a_{1}, \ldots, a_{m}\right)\right\rangle \leq \mathbf{D} \wedge\left\langle t_{i}^{\mathbf{A}}\left(a_{1}, \ldots, a_{m}\right)\right\rangle \leq$ $(\mathbf{D} \wedge \mathbf{B}) \vee(\mathbf{D} \wedge \mathbf{C})$ for $i=1, \ldots, n$. Thus $d \in(\mathbf{D} \wedge \mathbf{B}) \vee(\mathbf{D} \wedge \mathbf{C})$.

The condition of Theorem 1.4 always holds for unary operations, so we have a well-known result that unary algebras have distributive subalgebra lattices. Next, for binary operations it is sufficient to verify this condition for different elements only. In general, we cannot restrict our attention to $b=k^{\mathbf{A}}\left(a_{1}, \ldots, a_{\kappa(k)}\right)$ in Theorem 1.4 (e.g. we can take this six-element quasigroup from [3]).

\section{Auxiliary Results}

Here we prove technical results which will be useful in the next section to construct a two-generated non-cyclic quasigroup with a distributive subquasigroup lattice. In our construction we use partial algebras (basic concepts and 
facts concerning partial algebras can be found, for example, in [1]). Beside usual subalgebras defined in the same way as in the total case, we need here concepts of weak and relative (partial) subalgebras.

Let $\mathbf{A}=\left(A,\left(k^{\mathbf{A}}\right)_{k \in K}\right)$ and $\mathbf{B}=\left(B,\left(k^{\mathbf{B}}\right)_{k \in K}\right)$ be partial algebras of the same type. We say that $\mathbf{B}$ is a weak subalgebra of $\mathbf{A}$ if $B \subseteq A$ and for each $k \in K, k^{\mathbf{B}} \subseteq k^{\mathbf{A}}$. A weak subalgebra $\mathbf{B}$ of $\mathbf{A}$ is said to be relative if $k^{\mathbf{B}}=k^{\mathbf{A}} \cap\left(B^{\kappa(k)} \times B\right)$ for all $k \in K$.

Lemma 2.1. Let $\left(\mathbf{A}_{i}\right)_{i=0}^{\infty}$ be a sequence of partial algebras (of the same type) such that $\mathbf{A}_{i}$ is a weak (relative) subalgebra of $\mathbf{A}_{i+1}$ for $i \in \mathbb{N}$ and let $a \in$ $\bigcup_{i=0}^{\infty} A_{i}$. Then

(a) $\bigcup_{i=0}^{\infty} \mathbf{A}_{i}=\left(\bigcup_{i=0}^{\infty} A_{i},\left(\bigcup_{i=0}^{\infty} k^{\mathbf{A}_{i}}\right)_{k \in K}\right)$ is a well-defined partial algebra which contains algebras $\left\{\mathbf{A}_{i}\right\}_{i=0}^{\infty}$ as weak (relative) subalgebras.

(b) For each $b \in\langle a\rangle_{\bigcup_{i=0}^{\infty}} \mathbf{A}_{i}$, there is $l \in \mathbb{N}$ such that $b \in\langle a\rangle_{\mathbf{A}_{l}}$.

Proof. ( $a$ ) is obvious. To see (b) take $m \in \mathbb{N}$ such that $a \in A_{m}$ and let $b \in\langle a\rangle_{\mathbf{A}}$, where $\mathbf{A}=\bigcup_{i=0}^{\infty} \mathbf{A}_{i}$. Similarly as for total algebras, there is a term $t(x)$ such that $a \in \operatorname{dom}\left(t^{\mathbf{A}}\right)$ and $t^{\mathbf{A}}(a)=b$. If $t(x)=x$, then $b=a \in\langle a\rangle_{\mathbf{A}_{m}}$. Thus it is sufficient to apply induction on length of $t(x)=f\left(t_{1}(x), \ldots, t_{n}(x)\right)$, because if $t_{i}^{\mathbf{A}}(a) \in\langle a\rangle_{\mathbf{A}_{j_{i}}}$ for $i=1, \ldots, n$, then $t_{1}^{\mathbf{A}}(a), \ldots, t_{n}^{\mathbf{A}}(a) \in\langle a\rangle_{\mathbf{A}_{k}}$ where $k=\max \left\{j_{1}, \ldots, j_{n}\right\}$.

Lemma 2.2. Let $\left(\mathbf{A}_{i}\right)_{i=0}^{\infty}$ be a sequence of partial algebras (of the same type) such that for each $i \in \mathbb{N}, \mathbf{A}_{i}$ has a distributive subalgebra lattice and $\mathbf{A}_{i}$ is a weak subalgebra of $\mathbf{A}_{i+1}$. Then $\bigcup_{i=0}^{\infty} \mathbf{A}_{i}$ has also a distributive subalgebra lattice.

Proof. Take an $n$-ary operation $f^{\mathbf{A}}$ (where $\left.n \geq 2\right),\left(a_{1}, \ldots, a_{n}\right) \in \operatorname{dom}\left(f^{\mathbf{A}}\right)$ and $b \in\left\langle f^{\mathbf{A}}\left(a_{1}, \ldots, a_{n}\right)\right\rangle_{\mathbf{A}}$. Then there is $i \in \mathbb{N}$ such that $\left(a_{1}, \ldots, a_{n}\right) \in$ $\operatorname{dom}\left(f^{\mathbf{A}_{i}}\right)$. Next, by Lemma 2.1(b), $b \in\left\langle f^{\mathbf{A}}\left(a_{1}, \ldots, a_{n}\right)\right\rangle_{\mathbf{A}_{j}}$ for some $j \in \mathbb{N}$. Taking $k=\max \{i, j\}$ we have $b \in\left\langle f^{\mathbf{A}_{k}}\left(a_{1}, \ldots, a_{n}\right)\right\rangle_{\mathbf{A}_{k}}$. Hence and by Theorem 1.4, b belongs to $\left(\langle b\rangle_{\mathbf{A}_{k}} \wedge\left\langle a_{1}\right\rangle_{\mathbf{A}_{k}}\right) \vee\left(\langle b\rangle_{\mathbf{A}_{k}} \wedge\left\langle a_{2}\right\rangle_{\mathbf{A}_{k}}\right) \vee \ldots \vee\left(\langle b\rangle_{\mathbf{A}_{k}} \wedge\left\langle a_{n}\right\rangle_{\mathbf{A}_{k}}\right)$ which is contained in $\left(\langle b\rangle_{\mathbf{A}} \wedge\left\langle a_{1}\right\rangle_{\mathbf{A}}\right) \vee\left(\langle b\rangle_{\mathbf{A}} \wedge\left\langle a_{2}\right\rangle_{\mathbf{A}}\right) \vee \ldots \vee\left(\langle b\rangle_{\mathbf{A}} \wedge\left\langle a_{n}\right\rangle_{\mathbf{A}}\right)$. So $\mathbf{A}$ satisfies the condition of Theorem 1.4.

Lemma 2.3. Let a partial algebra $\mathbf{A}$ with binary operations $g_{1}^{\mathbf{A}}, g_{2}^{\mathbf{A}}, \ldots, g_{n}^{\mathbf{A}}$ and unary operations $\varphi_{1}^{\mathbf{A}}, \varphi_{2}^{\mathbf{A}}, \varphi_{3}^{\mathbf{A}}$.. satisfy the following conditions:

(*) for $a, b \in A$ and $i=1, \ldots, n$, if $(a, b) \in \operatorname{dom}\left(g_{i}^{\mathbf{A}}\right)$, then $b \in\left\langle a, g_{i}^{\mathbf{A}}(a, b)\right\rangle_{\mathbf{A}}$ and $a \in\left\langle b, g_{i}^{\mathbf{A}}(a, b)\right\rangle_{\mathbf{A}}$,

(**) $\mathbf{A}$ is non-cyclic and generated by two elements $x, y$.

Then there is a partial algebra $\mathbf{B}$ with binary operations $g_{1}^{\mathbf{B}}, g_{2}^{\mathbf{B}}, \ldots, g_{n}^{\mathbf{B}}$, unary operations $\varphi_{1}^{\mathbf{B}}, \varphi_{2}^{\mathbf{B}}, \varphi_{3}^{\mathbf{B}} \ldots$ and with additional unary operations $f_{1}^{\mathbf{B}}, f_{2}^{\mathbf{B}}, f_{3}^{\mathbf{B}}, \ldots$, which satisfies the following conditions:

(a) $\mathbf{A}$ is a relative subalgebra of $\mathbf{B}$ such that $\operatorname{dom}\left(g_{i}^{\mathbf{B}}\right)=\operatorname{dom}\left(g_{i}^{\mathbf{A}}\right)$ for $i=$ $2,3, \ldots, n$ and $\operatorname{dom}\left(\varphi_{j}^{\mathbf{B}}\right)=\operatorname{dom}\left(\varphi_{j}^{\mathbf{A}}\right)$ for $j=1,2,3, \ldots$ 
(b) $\mathbf{B}$ has a distributive subalgebra lattice.

(c) $\mathbf{B}$ is non-cyclic and generated by $x, y$.

(d) B satisfies also $(*)$.

Proof. Let $F=\left\{f_{1}, f_{2}, f_{3}, \ldots\right\}$ and let $F_{1}, F_{2}, F_{3}, \ldots$ be a partition of $F$ into infinite subsets.

We first construct some sequence of algebras $\left(\mathbf{A}_{i}\right)_{i=0}^{\infty}$. Take $\mathbf{A}_{0}=\mathbf{A}$ (which has at most countable many elements) and assume that we have constructed $\mathbf{A}_{k-1}$ which is also at most countable. Let $\mathcal{T}_{k}$ be a family of all triples $(a, b, c)$ of pairwise distinct elements from $\mathbf{A}_{k-1}$ for which there is $1 \leq i \leq n$ such that $(a, b) \in \operatorname{dom}\left(g_{i}^{\mathbf{A}_{k-1}}\right), g_{i}^{\mathbf{A}_{k-1}}(a, b) \notin\{a, b\}$ and $c \in\left\langle g_{i}^{\mathbf{A}_{k-1}}(a, b)\right\rangle_{\mathbf{A}_{k-1}}$. $\mathcal{T}_{k}$ is at most countable (since $\mathbf{A}_{k-1}$ is at most countable) and $F_{k}$ is countable, so we can assign to each triple $(a, b, c) \in \mathcal{T}_{k}$ two distinct unary operation symbols $f_{a b c}^{1}$ and $f_{a b c}^{2}$ from $F_{k}$, in such a way that distinct triples have distinct operation symbols. We define $\mathbf{A}_{k}$ in the following way: First, for each $(a, b, c) \in \mathcal{T}_{k}$ we add to $\mathbf{A}_{k-1}$ two new distinct elements $d_{a b c}, e_{a b c}$, outside $\mathbf{A}_{k-1}$ and distinct for distinct triples. Secondly, we set $\operatorname{dom}\left(f_{a b c}^{1}\right)=\{a, c\}$, $f_{a b c}^{1}(a)=d_{a b c}=f_{a b c}^{1}(c)$ and $\operatorname{dom}\left(f_{a b c}^{2}\right)=\{b, c\}, f_{a b c}^{2}(b)=e_{a b c}=f_{a b c}^{2}(c)$. Thirdly, we extend $g_{1}^{\mathbf{A}_{k-1}}$ by setting $g_{1}^{\mathbf{A}_{k}}\left(d_{a b c}, e_{a b c}\right)=g_{1}^{\mathbf{A}_{k}}\left(e_{a b c}, d_{a b c}\right)=c$.

Let $\mathbf{B}=\bigcup_{i=0}^{\infty} \mathbf{A}_{i}$. Obviously $\mathbf{B}$ satisfies $(a)$, and also $\mathbf{A}_{0}, \mathbf{A}_{1}, \mathbf{A}_{2}, \ldots$ are relative subalgebras of $\mathbf{B}$.

For all $a, b \in A_{k+1},(a, b) \in \operatorname{dom}\left(g_{1}^{\mathbf{A}_{k+1}}\right)$ implies $g_{1}^{\mathbf{A}_{k+1}}(a, b) \in \mathbf{A}_{k}$, and if additionally $a, b \in A_{k}$, then $(a, b) \in \operatorname{dom}\left(g_{1}^{\mathbf{A}_{k}}\right)$. Hence, if $a, b \in A_{k}$ and $(a, b) \in \operatorname{dom}\left(g_{1}^{\mathbf{B}}\right)$, then $(a, b) \in \operatorname{dom}\left(g_{1}^{\mathbf{A}_{k}}\right)$.

$$
\langle v\rangle_{\mathbf{A}_{k+1}} \wedge \mathbf{A}_{k}=\langle v\rangle_{\mathbf{A}_{k}} \quad \text { for each } v \in A_{k} .
$$

It is sufficient to prove $\subseteq$. Let $X$ be the carrier of $\langle v\rangle_{\mathbf{A}_{k}}$. By the above fact, $X$ is closed in $\mathbf{A}_{k+1}$ under all binary operations. Take the set $Y$ of all elements $z \in A_{k+1} \backslash A_{k}$ such that $z$ is a value of some unary operation of $\mathbf{A}_{k+1}$ on some element from $X$. Since none unary operation of $\mathbf{A}_{k+1}$ is defined on any element from $A_{k+1} \backslash A_{k}, X \cup Y$ is closed under all unary operations. This set is also closed under $g_{2}^{\mathbf{A}_{k+1}}, \ldots, g_{n}^{\mathbf{A}_{k+1}}$. So take $(u, w) \in \operatorname{dom}\left(g_{1}^{\mathbf{A}_{k+1}}\right)$ and $u, w \in X \cup Y$. First, $g_{1}^{\mathbf{A}_{k+1}}$ is not defined on any pair with one element in $\mathbf{A}_{k}$ and the other outside. Hence, $u, w \in X$ or $u, w \in Y$. If the first case holds, then $g_{1}^{\mathbf{A}_{k+1}}(u, w)=g_{1}^{\mathbf{A}_{k}}(u, w) \in X$. Thus let $u, w \in Y$. Then first, $u \neq w$. Secondly, $u$ and $w$ are images of elements $z_{1}, z_{2} \in X$ respectively, under some unary operations. Thirdly, by the definition of $g^{\mathbf{A}_{k+1}}$, there is exactly one $(a, b, c) \in \mathcal{T}_{k}$ such that $c=g_{1}^{\mathbf{A}_{k+1}}(u, w) \in\langle a, b\rangle_{\mathbf{A}_{k}}$ and $f_{a b c}^{1}(a)=u, f_{a b c}^{2}(b)=w$ and $f_{a b c}^{1}(c)=u, f_{a b c}^{2}(c)=w$. Our construction implies $z_{1}, z_{2} \in\{a, b, c\}$. If $z_{1}=c$ or $z_{2}=c$, then $g_{1}^{\mathbf{A}_{k+1}}(u, w)=c \in\left\{z_{1}, z_{2}\right\} \subseteq X$. If $\left\{z_{1}, z_{2}\right\}=\{a, b\}$, then $a, b \in X$, so $c \in\langle a, b\rangle_{\mathbf{A}_{k}} \subseteq X$. Cases $z_{1}=a=z_{2}$ and $z_{1}=b=z_{2}$ cannot hold, because $(u, w) \in \operatorname{dom}\left(g^{\mathbf{A}_{k+1}}\right)$. 
None of $\mathbf{A}_{0}, \mathbf{A}_{1}, \ldots$ is cyclic. Otherwise we would take the least $k$ such that $\mathbf{A}_{k}$ has one generator $v . k \geq 1$, so $v \in A_{k-1}$, because $\langle w\rangle_{\mathbf{A}_{k}}=\{w\}$ for $w \in A_{k} \backslash A_{k-1}$. By (eq), $v$ generates $\mathbf{A}_{k-1}$, a contradiction.

$x, y$ generate all algebras $\mathbf{A}_{k}$, so $\mathbf{B}=\langle x, y\rangle_{\mathbf{B}}$. $\mathbf{B}$ is non-cyclic. Assume otherwise that $b$ generates B. Then $x, y \in\langle b\rangle_{\mathbf{B}}$. By Lemma 2.1(b) there are $i$ and $j$ such that $x \in\langle b\rangle_{\mathbf{A}_{i}}$ and $y \in\langle b\rangle_{\mathbf{A}_{j}}$. Hence, $x, y \in\langle b\rangle_{\mathbf{A}_{k}}$ for $k=\max \{i, j\}$. Thus $\mathbf{A}_{k}=\langle b\rangle_{\mathbf{A}_{k}}$, a contradiction.

$\mathbf{B}$ satisfies $(*)$. Take $g_{i}^{\mathbf{B}}$, a pair $(u, w) \in \operatorname{dom}\left(g_{i}^{\mathbf{B}}\right)$ and let $v=g_{i}^{\mathbf{B}}(u, w)$. If $i \geq 2$, then $(u, w) \in \operatorname{dom}\left(g_{i}^{\mathbf{A}}\right)$ and $g_{i}^{\mathbf{A}}(u, w)=g_{i}^{\mathbf{B}}(u, w)$. Hence, $w \in\langle u, v\rangle_{\mathbf{A}} \subseteq$ $\langle u, v\rangle_{\mathbf{B}}$ and $u \in\langle w, v\rangle_{\mathbf{A}} \subseteq\langle w, v\rangle_{\mathbf{B}}$. Thus take $i=1$ and the least number $k$ such that $u, w \in A_{k}$. Then $(u, w) \in \operatorname{dom}\left(g_{1}^{\mathbf{A}_{k}}\right)$ and $v \in A_{k}$. If $k=0$, then similarly as for $i \geq 2$ we obtain required conditions. If $k \geq 1$, then there are two unary operations $f^{\mathbf{A}_{k}}, h^{\mathbf{A}_{k}}$ defined on $v$ and $f^{\mathbf{A}_{k}}(v)=u, h^{\mathbf{A}_{k}}(v)=w$. Hence, $u, w \in\langle v\rangle_{\mathbf{A}_{k}} \subseteq\langle v\rangle_{\mathbf{B}}$.

We use Theorem 1.4 to show that $\mathbf{B}$ has a distributive subalgebra lattice. Take $g_{i}^{\mathbf{B}}$ and $(u, w) \in \operatorname{dom}\left(g_{i}^{\mathbf{B}}\right)$. Let $v \in\left\langle g_{i}^{\mathbf{B}}(u, w)\right\rangle_{\mathbf{B}}$. Then $v \in\left\langle g_{i}^{\mathbf{B}}(u, w)\right\rangle_{\mathbf{A}_{k}}$ for some $k$. We can also assume $u, w \in A_{k}$. $\mathbf{A}_{k}$ is a relative subalgebra of $\mathbf{B}$, so $g_{i}^{\mathbf{A}_{k}}(u, w)$ is defined and equal $g_{i}^{\mathbf{B}}(u, w)$. Thus $v \in\left\langle g_{i}^{\mathbf{A}_{k}}(u, w)\right\rangle_{\mathbf{A}_{k}}$ (if $i \geq 2$, then $u, w \in A$, but $v$ need not be in $\mathbf{A}$ ). Then there are two unary operations $f^{\mathbf{A}_{k+1}}, h^{\mathbf{A}_{k+1}}$ of $\mathbf{A}_{k+1}$ defined on $u, w$ respectively, such that $\left(f^{\mathbf{A}_{k+1}}(u), h^{\mathbf{A}_{k+1}}(w)\right) \in \operatorname{dom}\left(g^{\mathbf{A}_{k+1}}\right)$ and $g^{\mathbf{A}_{k+1}}\left(f^{\mathbf{A}_{k+1}}(u), h^{\mathbf{A}_{k+1}}(w)\right)=v$ and $f^{\mathbf{A}_{k+1}}(v)=f^{\mathbf{A}_{k+1}}(u) \in\langle u\rangle_{\mathbf{A}_{k+1}} \cap\langle v\rangle_{\mathbf{A}_{k+1}}$ and $h^{\mathbf{A}_{k+1}}(v)=h^{\mathbf{A}_{k+1}}(w) \in$ $\langle u\rangle_{\mathbf{A}_{k+1}} \cap\langle v\rangle_{\mathbf{A}_{k+1}}$. In other words, $v \in\left(\langle u\rangle_{\mathbf{A}_{k+1}} \wedge\langle v\rangle_{\mathbf{A}_{k+1}}\right) \vee\left(\langle w\rangle_{\mathbf{A}_{k+1}} \wedge\right.$ $\left.\langle v\rangle_{\mathbf{A}_{k+1}}\right) \subseteq\left(\langle u\rangle_{\mathbf{B}} \wedge\langle v\rangle_{\mathbf{B}}\right) \vee\left(\langle w\rangle_{\mathbf{B}} \wedge\langle v\rangle_{\mathbf{B}}\right)$.

Now we construct a two-generated commutative groupoid $\mathcal{G}$ (i.e. a set with one commutative binary operation) with a distributive subgroupoid lattice, which satisfies (iv) of Theorem 1.1 (i.e. for every $a, b,\langle a, b\rangle_{\mathcal{G}}=\langle a, a b\rangle_{\mathcal{G}}=$ $\left.\langle b, a b\rangle_{\mathcal{G}}\right)$, but $\mathcal{G}$ is non-cyclic.

Take a set of unary operation symbols $F=\left\{f_{0}, f_{1}, f_{2}, \ldots\right\}$. Let $F_{\text {od }}=$ $\left\{f_{1}, f_{3}, f_{5}, \ldots\right\}$ and $F_{e v}=\left\{f_{0}, f_{2}, f_{4}, \ldots\right\}$. Next, we divide $F_{o d}$ into pairwise disjoint infinite subsets $F_{0}, F_{1}, F_{2}, \ldots$.

Further, for each $E \subseteq F$, let $\bar{E}$ be a set of all $f_{k} \in F$ such that $f_{k} \in E$ or $f_{k-1}, f_{k+1} \in E$ (if $k=0$, then $f_{0} \in E$ or $f_{1} \in E$ ). It is a closure (and even algebraic) operator on $F$.

We first construct a sequence $\left(\mathbf{A}_{k}\right)_{k=0}^{\infty}$ of partial algebras with one binary operation $g$ and unary operations $f_{0}, f_{1}, f_{2}, \ldots$ such that for $k \in \mathbb{N}, \mathbf{A}_{k}$ is a relative subalgebra of $\mathbf{A}_{k+1}$ and $g^{\mathbf{A}_{k}}$ is commutative (i.e. if $(a, b) \in \operatorname{dom}\left(g^{\mathbf{A}_{k}}\right)$, then $(b, a) \in \operatorname{dom}\left(g^{\mathbf{A}_{k}}\right)$ and $\left.g^{\mathbf{A}_{k}}(b, a)=g^{\mathbf{A}_{k}}(a, b)\right)$. Moreover, $\mathbf{A}_{k}$ has a distributive subalgebra lattice, is non-cyclic and generated by two elements. Additionally, this sequence satisfies the following technical conditions:

(g1) for all elements $a, b$ of $\mathbf{A}_{k}, \quad(a, b) \notin \mathcal{E}_{k-1} \Longleftrightarrow(a, b) \in \operatorname{dom}\left(g^{\mathbf{A}_{k}}\right)$, $\mathcal{E}_{k}$ is the set of all pairs $(a, b)$ such that $a=b$ or $(a, b) \in \mathcal{X}_{k}$ or $(b, a) \in \mathcal{X}_{k}$, 
where $\mathcal{X}_{k}$ is the set of all pairs $(a, b)$ such that $a \neq b, a \in \operatorname{dom}\left(f^{\mathbf{A}_{k}}\right)$ and $f^{\mathbf{A}_{k}}(a)=b$ for some $f \in F$.

(g2) for all $f \in \overline{F_{0} \cup F_{1} \cup \cdots \cup F_{k-1}}, \quad A_{k-1} \subseteq \operatorname{dom}\left(f^{\mathbf{A}_{k}}\right)$, and if $f \neq \varphi$, then $f^{\mathbf{A}_{k-1}}(a) \neq \varphi^{\mathbf{A}_{k-1}}(a)$ for $a \in A_{k-1}$.

(g3) for each $i \in \mathbb{N}$ and $a \in A_{k-1}$, if $f_{i}, f_{i+1} \in \overline{F_{0} \cup F_{1} \cup \cdots \cup F_{k-1}}$, then

$$
\left(f_{i}^{\mathbf{A}_{k}}(a), f_{i+1}^{\mathbf{A}_{k}}(a)\right) \in \operatorname{dom}\left(g^{\mathbf{A}_{k}}\right) \text { and } g^{\mathbf{A}_{k}}\left(f_{i}^{\mathbf{A}_{k}}(a), f_{i+1}^{\mathbf{A}_{k}}(a)\right)=a,
$$

(g4) for all $a, b \in A_{k}$, if $(a, b) \in \operatorname{dom}\left(g^{\mathbf{A}_{k}}\right)$, then $b \in\left\langle a, g^{\mathbf{A}_{k}}(a, b)\right\rangle_{\mathbf{A}_{k}}$ and $a \in\left\langle b, g^{\mathbf{A}_{k}}(a, b)\right\rangle_{\mathbf{A}_{k}}$.

Let $\mathbf{A}=\left(\{x, y, z\}, g^{\mathbf{A}}\right)$ be a three-element algebra, where $g^{\mathbf{A}}$ is a binary operation such that $g^{\mathbf{A}}(x, y)=g^{\mathbf{A}}(y, x)=z, g^{\mathbf{A}}(x, z)=g^{\mathbf{A}}(z, x)=y, g^{\mathbf{A}}(y, z)=$ $g^{\mathbf{A}}(z, y)=x$, (precisely, these pairs belong to $\operatorname{dom}\left(g^{\mathbf{A}}\right)$ and all other pairs not). Let $\mathbf{A}_{0}$ be the extension of $\mathbf{A}$ from Lemma 2.3, where we take $F_{0}$ as the set of new unary operation symbols. Then $\mathbf{A}_{0}$ has a distributive subalgebra lattice, is non-cyclic and generated by $x, y$, and also satisfies (g4). $g^{\mathbf{A}_{0}}$ is commutative, and for each $(a, b) \in \mathcal{E}_{0},(a, b) \notin \operatorname{dom}\left(g^{\mathbf{A}_{0}}\right)$.

Assume that we have constructed algebras $\mathbf{A}_{0}, \mathbf{A}_{1}, \ldots, \mathbf{A}_{k-1}$, where $\overline{F_{0} \cup \cdots \cup F_{j-1}} \cup F_{j}$ is the set of all unary operations of $\mathbf{A}_{j}$ for $j=1, \ldots, k-1$, and $x, y$ generate these algebras. Additionally we assume

(i.1) For all $a \in A_{k-1} \backslash A_{k-2}, a \in \operatorname{dom}\left(f^{\mathbf{A}_{k-1}}\right)$ implies $f \in F_{k-1}$.

(i.2) For all $a \in A_{k-1}, f^{\mathbf{A}_{k-1}}(a) \neq \varphi^{\mathbf{A}_{k-1}}(a)$ for $f \neq \varphi$ (if both operations are defined on $a$ ).

(i.3) For each pair $(a, b)$ of elements of $\mathbf{A}_{k-1}$, there is at most one $c \in A_{k-1}$ and at most one $i=0,1,2, \ldots$ such that $c \in \operatorname{dom}\left(f_{i}^{\mathbf{A}_{k-1}}\right) \cap \operatorname{dom}\left(f_{i+1}^{\mathbf{A}_{k-1}}\right)$ and $f_{i}^{\mathbf{A}_{k-1}}(c)=a, f_{i+1}^{\mathbf{A}_{k-1}}(c)=b$.

$\mathbf{A}_{0}$ satisfies these conditions by the construction from Lemma 2.3.

Let $\mathcal{B}_{k}$ be the family of all undirected pairs $\{a, b\}$ of distinct elements such that $(a, b),(b, a) \notin \mathcal{E}_{k-1}$ and $(a, b) \notin \operatorname{dom}\left(g^{\mathbf{A}_{k-1}}\right)$. For each $\{a, b\} \in \mathcal{B}_{k}$ we add a new element $e_{a b}$ to $\mathbf{A}_{k-1}$. These elements are pairwise distinct and outside $\mathbf{A}_{k-1}$. An algebra $\mathbf{B}_{k}$ is obtained from $\mathbf{A}_{k-1}$ by extending $g^{\mathbf{A}_{k-1}}$ as follows: $g^{\mathbf{B}_{k}}(a, b)=g^{\mathbf{B}_{k}}(b, a)=e_{a b}, g^{\mathbf{B}_{k}}\left(e_{a b}, a\right)=g^{\mathbf{B}_{k}}\left(a, e_{a b}\right)=b, g^{\mathbf{B}_{k}}\left(e_{a b}, b\right)=$ $g^{\mathbf{B}_{k}}\left(b, e_{a b}\right)=a$.

Next, for each $a \in A_{k-1}$ and $f \in \overline{F_{0} \cup \cdots \cup F_{k-1}}$, if $a \notin \operatorname{dom}\left(f^{\mathbf{A}_{k-1}}\right)$, then we add to $\mathbf{B}_{k}$ a new element $f(a)$. As above they are pairwise distinct and outside $\mathbf{B}_{k}$. We extend $\mathbf{B}_{k}$ to an algebra $\mathbf{C}_{k}$, setting $f^{\mathbf{C}_{k}}(a)=f(a)$. $\mathbf{C}_{k}$ satisfies (i.2) and (i.3).

Let $i \in \mathbb{N}$ be such that $f_{i}, f_{i+1} \in \overline{F_{0} \cup \cdots \cup F_{k-1}}$. For any $a \in A_{k-1}$, let $u=f_{i}^{\mathbf{C}_{k}}(a), w=f_{i+1}^{\mathbf{C}_{k}}(a)$. If $(u, w) \notin \operatorname{dom}\left(g^{\mathbf{B}_{k}}\right)$ (thus also $\left.(w, u) \notin \operatorname{dom}\left(g^{\mathbf{B}_{k}}\right)\right)$, then, since $\mathbf{C}_{k}$ satisfies (i.3), we can extend $g^{\mathbf{B}_{k}}$ to a new operation $g^{\mathbf{C}_{k}}$, setting $g^{\mathbf{C}_{k}}(u, w)=g^{\mathbf{C}_{k}}(w, u)=a$.

$\mathbf{C}_{k}$ satisfies (g2), (g4) (since they hold for $\mathbf{A}_{k-1}$ ) and is generated by $x, y$. Next, for $Z \subseteq A_{k-1},\langle Z\rangle_{\mathbf{A}_{k}}=\langle Z\rangle_{\mathbf{C}_{k}} \cap \mathbf{A}_{k}$ and $\langle p\rangle_{\mathbf{C}_{k}}=\{p\}$ for $p \in C_{k} \backslash A_{k-1}$. Hence, $\mathbf{C}_{k}$ is not one-generated. 
Applying Lemma 2.3 to $\mathbf{C}_{k}$ and $F_{k}$, we obtain a non-cyclic two-generated algebra $\mathbf{A}_{k}$ with a distributive subalgebra lattice. $\mathbf{A}_{k-1}$ is a relative subalgebra of $\mathbf{A}_{k}$ and $g^{\mathbf{A}_{k}}$ is commutative. $\mathbf{A}_{k}$ satisfies (g2), (g4) (because $\mathbf{C}_{k}$ satisfies these two conditions) and (g1) (by the definition of $\mathbf{B}_{k}$ ). By the construction from the proof of Lemma 2.3 and induction hypotheses, (i.l) and (i.2) hold for $\mathbf{A}_{k}$.

Take $c \in A_{k}$ such that $c \in \operatorname{dom}\left(f_{i}^{\mathbf{A}_{k}}\right) \cap \operatorname{dom}\left(f_{i+1}^{\mathbf{A}_{k}}\right)$. Then $i$ or $i+1$ is even. The operation with even index belongs to $\overline{F_{0} \cup \cdots \cup F_{k-1}} \cup F_{k}$ and does not belong to $F_{k}$, so by the definition of the second operation belongs to $F_{0} \cup \cdots \cup F_{k-1}$. Hence, $\mathbf{A}_{k}$ satisfies (i.3), because $\mathbf{C}_{k}$ satisfies it.

$\mathbf{A}_{k}$ satisfies (g3). Let $f_{i}, f_{i+1} \in \overline{F_{0} \cup \cdots \cup F_{k-1}}$ and $a \in A_{k-1}$. If $a \in$ $A_{k-1} \backslash A_{k-2}$, then by (i.1), $f_{i}$ or $f_{i+1}$ is not defined on $a$ in $\mathbf{A}_{k-1}$, because $f_{i}$ or $f_{i+1}$ is in $F_{e v}$ (in particular, outside $F_{k-1}$ ). Hence we obtain (g3), because $u=$ $f_{i}^{\mathbf{C}_{k}}(a)$ or $w=f_{i+1}^{\mathbf{C}_{k}}(a)$ does not belong to $\mathbf{B}_{k}$, so $(u, w),(w, u) \notin \operatorname{dom}\left(g^{\mathbf{B}_{k}}\right)$. Let $a \in A_{k-2}$. Then by (g2) all operations from $\overline{F_{0} \cup \cdots \cup F_{k-2}}$ are defined on $a$ in $\mathbf{A}_{k-1}$. Hence, if $f_{i}, f_{i+1} \in \overline{F_{0} \cup \cdots \cup F_{k-2}}$, then (g3) holds by induction hypothesis. Otherwise, the operation with even index does not belong to $\overline{F_{0} \cup \cdots \cup F_{k-2}}$, and is also outside $F_{k-1}$ (this is obvious, if the operation with odd index belongs to $\overline{F_{0} \cup \cdots \cup F_{k-2}}$; if not, then it belongs to $F_{k-1}$, so our fact is implied by the definition of - ). Thus the operation with even index is not an operation of $\mathbf{A}_{k-1}$. Hence again, $u$ or $w$ does not belong to $\mathbf{B}_{k}$.

Let $\mathbf{A}=\bigcup_{k=0}^{\infty} \mathbf{A}_{k}$. Then $\mathbf{A}_{0}, \mathbf{A}_{1}, \mathbf{A}_{2}, \ldots$ are relative subalgebras of $\mathbf{A}$. By Lemma 2.2, A has a distributive subalgebra lattice. $\mathbf{A}$ is generated by $x, y$ and $\mathbf{A}$ is not one-generated (if $a$ would generate $\mathbf{A}$, then $x, y \in\langle a\rangle_{\mathbf{A}}$, so by Lemma $2.1, x, y \in\langle a\rangle_{\mathbf{A}_{k}}$ for some $k$, thus $a$ would have to generate $\mathbf{A}_{k}$, a contradiction). By (g2), operations $f_{0}^{\mathbf{A}}, f_{1}^{\mathbf{A}}, f_{2}^{\mathbf{A}}, \ldots$ are total, since $\cup_{k=0}^{\infty} \overline{F_{0} \cup F_{1} \cup \cdots \cup F_{k}}=E$. Next, for each $a \in A, f_{0}^{\mathbf{A}}(a), f_{1}^{\mathbf{A}}(a), f_{2}^{\mathbf{A}}(a), \ldots$ are pairwise distinct, and also distinct from $a$ (by our construction).

Take $(a, b) \in \operatorname{dom}\left(g^{\mathbf{A}}\right)$ and let $c=g^{\mathbf{A}}(a, b)$. Then $(a, b) \in \operatorname{dom}\left(g^{\mathbf{A}_{k}}\right)$ and $g^{\mathbf{A}_{k}}(a, b)=c$ for some $k$. By $(\mathrm{g} 4), a \in\langle b, c\rangle_{\mathbf{A}_{k}} \subseteq\langle b, c\rangle_{\mathbf{A}}$ and $b \in\langle a, c\rangle_{\mathbf{A}_{k}} \subseteq$ $\langle a, c\rangle_{\mathbf{A}}$. Thus $\mathbf{A}$ satisfies (iv) of Theorem 1.1.

Let $\mathcal{X}$ and $\mathcal{E}$ be families of all pairs of elements of $\mathbf{A}$ defined analogously as $\mathcal{X}_{k}$ and $\mathcal{E}_{k}$ for $\mathbf{A}_{k}$. Then $\mathcal{E}=\bigcup_{k=0}^{\infty} \mathcal{E}_{k}$. Since $\left(\mathbf{A}_{k}\right)_{k=0}^{\infty}$ is ascending, satisfies (g1), (g3) and $\mathcal{E}_{k} \subseteq \mathcal{E}_{k+1}$, we obtain that $\mathbf{A}$ satisfies analogous versions of (g1), (g3) (where we replace $\mathbf{A}_{k}, \mathcal{E}_{k}$, by $\mathbf{A}, \mathcal{E}$ ).

If $(a, b) \notin \operatorname{dom}\left(g^{\mathbf{A}}\right)$, then, in particular, $a \in\langle b\rangle_{\mathbf{A}}$ or $b \in\langle a\rangle_{\mathbf{A}}$. Thus we can extend $\mathbf{A}$ to a total algebra $\mathbf{B}$, setting $g^{\mathbf{B}}(a, b)=g^{\mathbf{B}}(b, a)=b$ in the first case, and $g^{\mathbf{B}}(a, b)=g^{\mathbf{B}}(b, a)=a$ in the second. $\mathbf{A}$ and $\mathbf{B}$ have the same subalgebras, so $\mathbf{B}$ has a distributive subalgebra lattice, is two-generated, non-cyclic and satisfies (iv) of Theorem 1.1.

We can also build a (total) commutative groupoid $\mathcal{G}=(A, \circ)$ with these four properties. Let $a \circ b=g^{\mathbf{A}}(a, b)$ for $(a, b) \in \operatorname{dom}\left(g^{\mathbf{A}}\right)$. Next, for $a \in A$, let $a_{i}=f_{i}^{\mathbf{A}}(a)$ for $i=0,1,2, \ldots$. Since $g^{\mathbf{A}}$ is not defined on $(a, a)$, we can set 
$a \circ a=a_{0}$. Since $\left(a, a_{0}\right) \notin \operatorname{dom}\left(g^{\mathbf{A}}\right)$, we can set $a \circ a_{0}=a_{0} \circ a=a_{1}$, and so on. $\circ$ is well-defined, because there are no $a, b \in A$ and $f, \varphi \in F$ such that $f^{\mathbf{A}}(a)=b$ and $\varphi^{\mathbf{A}}(b)=a$. This groupoid $\mathcal{G}$ and $\mathbf{A}$ have the same subalgebras. So $\mathcal{G}$ has a distributive subgroupoid lattice, $\mathcal{G}=\langle x, y\rangle_{\mathcal{G}}$ and $\mathcal{G}$ is not cyclic. By (g1), $\mathcal{G}$ is total. $\mathcal{G}$ satisfies also (iv) of Theorem 1.1 for each $(a, b) \in \operatorname{dom}\left(g^{\mathbf{A}}\right)$. Thus take $(a, b) \in \mathcal{E}^{\mathbf{A}}$ and $a \neq b$ (for $a=b$ it is obvious). Then there is $f_{i}^{\mathbf{A}}$ for some $i=0,1,2, \ldots$ such that $f_{i}^{\mathbf{A}}(a)=b$ or $f_{i}^{\mathbf{A}}(b)=a$. Assume that $b=f_{i}^{\mathbf{A}}(a)=a_{i}$ (the second case is analogous). Then $a \circ b=b \circ a=a_{i+1}$, so $b \in\langle a\rangle_{\mathcal{G}} \subseteq\left\langle a, a_{i+1}\right\rangle_{\mathcal{G}}$. By $(\mathrm{g} 3), b \circ a_{i+1}=g^{\mathbf{A}}\left(b, a_{i+1}\right)=a$, so also $a \in$ $\left\langle b, a_{i+1}\right\rangle_{\mathcal{G}}$.

\section{The Main Result}

Generalize the above construction we will build now a two-generated commutative quasigroup with a distributive subquasigroup lattice which is not cyclic.

We say that a binary (partial or total) operation $g^{\mathbf{A}}$ of an algebra $\mathbf{A}$ has a partial division property (in short, PDP) if for each $a, b \in A$, there is at most one $c \in A$ and at most one $d \in A$ such that $(a, c),(d, a) \in \operatorname{dom}\left(g^{\mathbf{A}}\right)$ and $g^{\mathbf{A}}(a, c)=b, g^{\mathbf{A}}(d, a)=b$. Similarly as for quasigroups, $g^{\mathbf{A}}$ defines two partial operations, the left $h_{1}^{\mathbf{A}}$ and the right $h_{2}^{\mathbf{A}}$ division (i.e. $h_{1}^{\mathbf{A}}(a, b)=c$ and $\left.h_{2}^{\mathbf{A}}(a, b)=d\right)$. If $g^{\mathbf{A}}$ is commutative, then $h_{1}^{\mathbf{A}}=h_{2}^{\mathbf{A}}$ (i.e. $\operatorname{dom}\left(h_{1}^{\mathbf{A}}\right)=\operatorname{dom}\left(h_{2}^{\mathbf{A}}\right)$ and $h_{1}^{\mathbf{A}}(u, w)=h_{2}^{\mathbf{A}}(u, w)$ for $\left.(u, w) \in \operatorname{dom}\left(h_{1}^{\mathbf{A}}\right)\right)$.

Take again a set of unary operation symbols $F=\left\{f_{0}, f_{1}, f_{2}, \ldots\right\}$ and let $F_{\text {od }}=\left\{f_{1}, f_{3}, f_{5}, \ldots\right\}, F_{e v}=\left\{f_{0}, f_{2}, f_{4}, \ldots\right\}$. We also divide $F_{o d}$ into pairwise disjoint infinite subsets $F_{0}, F_{1}, F_{2}, \ldots$. Recall that for $E \subseteq F, \bar{E}$ is the set of all $f_{k} \in F$ such that $f_{k} \in E$ or $f_{k-1}, f_{k+1} \in E$ (if $k=0$, then $f_{0} \in E$ or $f_{1} \in E$ ).

We first construct a sequence $\left(\mathbf{A}_{k}\right)_{k=0}^{\infty}$ of algebras such that for $k \in \mathbb{N}$, $\mathbf{A}_{k}$ has unary operations from the set $\overline{F_{0} \cup \cdots \cup F_{k-1}} \cup F_{k}$ and three binary operations: $g^{\mathbf{A}_{k}}$ being commutative and having PDP, $h^{\mathbf{A}_{k}}$ being the division operation associating with $g^{\mathbf{A}_{k}}$ and an auxiliary operation $u^{\mathbf{A}_{k}}$. Next, $\mathbf{A}_{k}$ is a non-cyclic two-generated algebra with a distributive subalgebra lattice, and also $\mathbf{A}_{k}$ is a relative subalgebra of $\mathbf{A}_{k+1}$. Further, the following technical conditions hold:

(q1) For all $\varphi \in \overline{F_{0} \cup \cdots \cup F_{k}}, \quad A_{k} \subseteq \operatorname{dom}\left(\varphi^{\mathbf{A}_{k+1}}\right)$, and if $f \neq \varphi$, then $f^{\mathbf{A}_{k}}(a) \neq \varphi^{\mathbf{A}_{k}}(a)$ for each $a \in A_{k}$.

(q2) For each $a, b \in A_{k}, \quad(a, b) \notin \mathcal{E}_{k} \Longleftrightarrow(a, b) \in \operatorname{dom}\left(g^{\mathbf{A}_{k+1}}\right)$,

recall $\mathcal{E}_{k}$ is the family of all pairs $(a, b)$ such that $a=b$ or $(a, b) \in \mathcal{X}_{k}$ or $(b, a) \in \mathcal{X}_{k}$, where $\mathcal{X}_{k}$ is the family of all pairs $(a, b)$ such that $a \neq b$ and $a \in \operatorname{dom}\left(f^{\mathbf{A}_{k}}\right), f^{\mathbf{A}_{k}}(a)=b$ for some $f \in F$.

(q3) For each $a, b \in A_{k}, \quad(a, b) \notin \mathcal{X}_{k} \cup \mathcal{Y}_{k}$ if and only if there is (exactly one) $c \in A_{k+1}$ such that $(a, c),(c, a) \in \operatorname{dom}\left(g^{\mathbf{A}_{k+1}}\right)$ and $g^{\mathbf{A}_{k+1}}(a, c)=$ $g^{\mathbf{A}_{k+1}}(c, a)=b$, 
where $\mathcal{Y}_{k}$ is the family of all pairs $(a, b)$ such that $a \neq b$ and there is $d \in A_{k}$ and there is $i \in \mathbb{N}$ such that $d \in \operatorname{dom}\left(f_{i}^{\mathbf{A}_{k}}\right) \cap \operatorname{dom}\left(f_{i+1}^{\mathbf{A}_{k}}\right)$ and $f_{i}^{\mathbf{A}_{k}}(d)=a, f_{i+1}^{\mathbf{A}_{k}}(d)=b$.

( $c$ is unique, because $g^{\mathbf{A}_{k}}$ has PDP. Note also that $a, b$ may be equal.)

(q4) For each $(a, b) \in \mathcal{Y}_{k}$, there is exactly one $d$ satisfying the conditions from the definition of $\mathcal{Y}_{k}$.

(q5) For all $a, b \in A_{k}$, if $(a, b) \in \mathcal{Y}_{k}$, then $(a, b) \notin \mathcal{X}_{k}$ and $(b, a) \notin \mathcal{X}_{k}$.

By $(\mathrm{q} 4), \mathcal{Y}_{k}$ define a binary operation $u^{\mathbf{A}_{k}}$ as follows: $\operatorname{dom}\left(u^{\mathbf{A}_{k}}\right)=\mathcal{Y}_{k}$ and $u^{\mathbf{A}_{k}}(a, b)=d$.

Let $\mathbf{A}=\left(\{x, y, z\}, g^{\mathbf{A}}\right)$, where $g^{\mathbf{A}}(x, y)=g^{\mathbf{A}}(y, x)=z$, and $g^{\mathbf{A}}$ is not defined on any other pair. $g^{\mathbf{A}}$ has PDP and the division corresponding to $g^{\mathbf{A}}$ equals $h^{\mathbf{A}}(x, z)=y, h^{\mathbf{A}}(y, z)=x$. A with $g^{\mathbf{A}}$ and $h^{\mathbf{A}}$, satisfies assumptions of Lemma 2.3, so (taking $F_{0}$ as the set of new unary operation symbols) we obtain an algebra $\mathbf{B}_{0}$ with binary operations $g^{\mathbf{B}_{0}}, h^{\mathbf{A}}$. The construction from Lemma 2.3 implies that $g^{\mathbf{B}_{0}}$ is commutative and has PDP. Thus $g^{\mathbf{B}_{0}}$ defines the division $h^{\mathbf{B}_{0}}$ which is an extension of $h^{\mathbf{A}}$. Let $\mathbf{A}_{0}$ be obtained from $\mathbf{B}_{0}$ by adding $h^{\mathbf{A}_{0}}=h^{\mathbf{B}_{0}}$ and taking the discrete (i.e. empty) operation $u^{\mathbf{A}_{0}}$. Only unary operations from $F_{0}$ (in particular with odd indices) are defined in $\mathbf{A}_{0}$, so $\mathcal{Y}_{0}=\emptyset$.

Take $(a, b) \in \operatorname{dom}\left(h^{\mathbf{A}_{0}}\right)$ and let $c=h^{\mathbf{A}_{0}}(a, b)$. In other words, $(a, c) \in$ $\operatorname{dom}\left(g^{\mathbf{A}_{0}}\right)$ and $g^{\mathbf{A}_{0}}(a, c)=b . a, c \in\{x, y\}$ implies $b=z$ and $h^{\mathbf{B}_{\mathbf{0}}}(a, b)=c=$ $h^{\mathbf{A}_{\mathbf{O}}}(a, b)$. If $a \notin\{x, y\}$ or $c \notin\{x, y\}$, then $a, c \notin\{x, y\}$ and $a, c \in\langle b\rangle_{\mathbf{B}_{0}}$. Hence, $\mathbf{A}_{0}$ and $\mathbf{B}_{0}=\left(B_{0}, g^{\mathbf{B}_{0}}, h^{\mathbf{A}}\right)$ have the same subalgebras. Thus $\mathbf{A}_{0}$ also has a distributive subalgebra lattice, two generators and is not cyclic.

Assume that we have constructed $\mathbf{A}_{0}, \mathbf{A}_{1}, \ldots, \mathbf{A}_{k}$. Assume also that for all $a, b \in A_{k}$,

(i.1) if $(a, b) \in \mathcal{E}_{k}$, then $(a, b),(b, a) \notin \operatorname{dom}\left(g^{\mathbf{A}_{k}}\right)$,

(i.2) if $(a, b) \in \mathcal{Y}_{k} \cup \mathcal{X}_{k}$, then there is no $c \in A_{k}$ such that $g^{\mathbf{A}_{k}}(a, c)=b$ (thus also $\left.g^{\mathbf{A}_{k}}(c, a)=b\right)$.

By the construction, $\mathbf{A}_{0}$ satisfies (i.1), (i.2) (note $\mathcal{Y}_{0}=\emptyset$ ).

For each pair $\{a, b\}$ of distinct elements such that $(a, b),(b, a) \notin \mathcal{X}_{k}$ and $(a, b) \notin \operatorname{dom}\left(g^{\mathbf{A}_{k}}\right)$, we add to $\mathbf{A}_{k}$ an element $e_{a b}$ (outside $\mathbf{A}_{k}$ and distinct for distinct pairs). We extend $\mathbf{A}_{k}$ to an algebra $\mathbf{B}_{k}$, setting $g^{\mathbf{B}_{k}}(a, b)=g^{\mathbf{B}_{k}}(b, a)=$ $e_{a b}$. Since $g^{\mathbf{B}_{k}}$ has PDP, the division $h^{\mathbf{A}_{k}}$ can be also extended to $h^{\mathbf{B}_{k}}$.

Let $\mathcal{A}_{k}$ be the family of all pairs $(a, b)$ of elements (not necessary distinct) of $\mathbf{A}_{k}$ such that $(a, b) \notin \mathcal{Y}_{k} \cup \mathcal{X}_{k}$ and there is no $c \in A_{k}$ such that $g^{\mathbf{A}_{k}}(a, c)=b$ (since $a, b \in A_{k}$, there is no such $c$ also in $\mathbf{B}_{k}$ ). We extend $\mathbf{B}_{k}$ to a new algebra $\mathbf{C}_{k}$ as follows: For each $(a, b) \in \mathcal{A}_{k}$ we add a new element $d_{a b}$ (outside $\mathbf{B}_{k}$ and distinct for distinct pairs) and set $g^{\mathbf{C}_{k}}\left(a, d_{a b}\right)=g^{\mathbf{C}_{k}}\left(d_{a b}, a\right)=b$. Then $g^{\mathbf{C}_{k}}$ has PDP, so we can also extend the division $h^{\mathbf{B}_{k}}$ to $h^{\mathbf{C}_{k}}$. Thus $\mathbf{C}_{k}$ satisfies (*) of Lemma 2.3. 
For each $a \in A_{k}$ and $f \in \overline{F_{0} \cup \cdots \cup F_{k}}$, if $a \notin \operatorname{dom}\left(f^{\mathbf{A}_{k}}\right)$ (so also $a \notin$ $\operatorname{dom}\left(f^{\mathbf{C}_{k}}\right)$ ), then we add to $\mathbf{C}_{k}$ an element $f(a)$ (these elements are pairwise distinct and outside $\mathbf{C}_{k}$ ) and we set $f^{\mathbf{D}_{k}}(a)=f(a)$ to obtain a new algebra $\mathbf{D}_{k}$. Then first, $\mathbf{A}_{k}$ is a relative subalgebra of $\mathbf{D}_{k}$. Secondly, $\mathbf{D}_{k}$ is generated by $x$ and $y$. Thirdly, similarly as in the construction of groupoid, we obtain that $\mathbf{D}_{k}$ is not cyclic. Fourthly, $\mathbf{D}_{k}$ satisfies also $(*)$ of Lemma 2.3.

Let $\mathcal{Z}_{k}$ be the family of all pairs $(a, b)$ of distinct elements of $\mathbf{D}_{k}$ for which there are $i \in \mathbb{N}$ and $c \in D_{k}$ such that $c \in \operatorname{dom}\left(f_{i}^{\mathbf{D}_{k}}\right) \cap \operatorname{dom}\left(f_{i+1}^{\mathbf{D}_{k}}\right)$ and $f_{i}^{\mathbf{D}_{k}}(c)=a, f_{i+1}^{\mathbf{D}_{k}}(c)=b$. Then $\mathcal{Y}_{k} \subseteq \mathcal{Z}_{k}$. Since $\mathbf{A}_{k}$ satisfies (q4), $\mathbf{D}_{k}$ also does it. Thus $\mathcal{Z}_{k}$ define an operation $u^{\mathbf{D}_{k}}$ being an extension of $u^{\mathbf{A}_{k}}$.

Applying Lemma 2.3 to $\mathbf{D}_{k}$ (with $g^{\mathbf{D}_{k}}=g^{\mathbf{C}_{k}}, h^{\mathbf{D}_{k}}=h^{\mathbf{C}_{k}}, u^{\mathbf{D}_{k}}$ and unary operations $f^{\mathbf{D}_{k}}$ for $f \in \overline{F_{0} \cup \cdots \cup F_{k}}$ ) and the set $F_{k+1}$ of new unary operation symbols, we obtain a non-cyclic two-generated (by $x, y$ ) algebra $\mathbf{A}_{k+1}$ with a distributive subalgebra lattice. $g^{\mathbf{A}_{k+1}}$ is commutative, has PDP (since $g^{\mathbf{C}_{k}}$ has it) and extends $g^{\mathbf{D}_{k}}$. Thus $g^{\mathbf{A}_{k+1}}$ defines the division $h^{\mathbf{A}_{k+1}}$ which extends $h^{\mathbf{D}_{k}}$.

By definitions of algebras $\mathbf{B}_{k}, \mathbf{C}_{k}, \mathbf{D}_{k}$ we have that (q1) and implications $\Longrightarrow$ in (q2) and (q3) hold for $\mathbf{A}_{k+1}$. Using additionally (i.1), (i.2) (for $\mathbf{A}_{k}$ ) and the construction from Lemma 2.3, inverse implications $\Longleftarrow$ in (q2) and (q3), and also (i.1) for $\mathbf{A}_{k+1}$, are easily obtained.

Take $c \in A_{k+1}$ such that $c \in \operatorname{dom}\left(f_{i}^{\mathbf{A}_{k+1}}\right) \cap \operatorname{dom}\left(f_{i+1}^{\mathbf{A}_{k+1}}\right)$ for some $i$. Then $i$ or $i+1$ is even. The operation with even index belongs to $\overline{F_{0} \cup \cdots \cup F_{k}} \cup F_{k+1}$ and does not belong to $F_{k+1}$, so by the definition of - , the second operation belongs to $F_{0} \cup \cdots \cup F_{k}$. Thus $\mathcal{Y}_{k+1}=\mathcal{Z}_{k}$. Hence and by induction hypotheses, $\mathbf{A}_{k+1}$ satisfies (q4), (q5) and also (i.2). Next, $\operatorname{dom}\left(u^{\mathbf{A}_{k+1}}\right)=\operatorname{dom}\left(u^{\mathbf{D}_{k}}\right)$ and $u^{\mathbf{A}_{k+1}}=u^{\mathbf{D}_{k}}$, where $u^{\mathbf{A}_{k+1}}$ is the operation defined by (q4). This completes the induction step.

$\left(\mathbf{A}_{k}\right)_{k=0}^{\infty}$ is a sequence of relative subalgebras, so $\mathcal{X}_{k} \subseteq \mathcal{X}_{k+1}, \mathcal{E}_{k} \subseteq \mathcal{E}_{k+1}$ and $\mathcal{Y}_{k} \subseteq \mathcal{Y}_{k+1}$ for $k \in \mathbb{N}$.

Let $\mathbf{A}=\bigcup_{k=0}^{\infty} \mathbf{A}_{k}$. By Lemma 2.2, $\mathbf{A}$ has a distributive subalgebra lattice. Similarly as in the construction of groupoid, we can show that $\mathbf{A}$ is noncyclic and generated by $x, y$. Since $g^{\mathbf{A}}=\bigcup_{k=0}^{\infty} g^{\mathbf{A}_{k}}$ and $g^{\mathbf{A}_{0}}, g^{\mathbf{A}_{1}}, g^{\mathbf{A}_{2}}, \ldots$ have PDP and $g^{\mathbf{A}_{k}} \subseteq g^{\mathbf{A}_{k+1}}$ for $k=0,1,2, \ldots$, it is easy to see that $g^{\mathbf{A}}$ also has PDP and the division corresponding to $g^{\mathbf{A}}$ equals $h^{\mathbf{A}}=\bigcup_{k=0}^{\infty} h^{\mathbf{A}_{k}}$. By (q1), $f_{0}^{\mathbf{A}}, f_{1}^{\mathbf{A}}, f_{2}^{\mathbf{A}}, \ldots$ are total. Moreover, $a, f_{0}^{\mathbf{A}}(a), f_{1}^{\mathbf{A}}(a), f_{2}^{\mathbf{A}}(a), \ldots$ are pairwise different for each $a \in A$.

Let $\mathcal{X}, \mathcal{E}$ and $\mathcal{Y}$ be families of all pairs of elements of $\mathbf{A}$ defined analogously as $\mathcal{X}_{k}, \mathcal{E}_{k}, \mathcal{Y}_{k}$ for $\mathbf{A}_{k}$. Then $\mathcal{X}=\bigcup_{k=0}^{\infty} \mathcal{X}_{k}, \mathcal{E}=\bigcup_{k=0}^{\infty} \mathcal{E}_{k}$ and $\mathcal{Y}=$ $\bigcup_{k=1}^{\infty} \mathcal{Y}_{k}$. Since $\left(\mathbf{A}_{k}\right)_{k=0}^{\infty}$ is ascending, satisfies (q2)-(q5) and $\mathcal{X}_{k} \subseteq \mathcal{X}_{k+1}, \mathcal{E}_{k} \subseteq$ $\mathcal{E}_{k+1}, \mathcal{Y}_{k} \subseteq \mathcal{Y}_{k+1}$, we obtain that $\mathbf{A}$ satisfies analogous versions of (q2)-(q5) (where we replace $\mathbf{A}_{k}, \mathcal{X}_{k}, \mathcal{E}_{k}, \mathcal{Y}_{k}$ by $\mathbf{A}, \mathcal{X}, \mathcal{E}, \mathcal{Y}$ ).

For each $a \in A$, let $c_{k}^{a}=f_{k}^{\mathbf{A}}(a)$ for $k=0,1,2, \ldots$, and let $c_{-1}^{a}=a$. By $(q 2),(a, a) \notin \operatorname{dom}\left(g^{\mathbf{A}}\right)$ and $\left(a, c_{k}^{a}\right),\left(c_{k}^{a}, a\right) \notin \operatorname{dom}\left(g^{\mathbf{A}}\right)$ for $k=0,1,2, \ldots$ 
Thus similarly as in the previous construction we can extend $g^{\mathbf{A}}$ to a new operation $\circ$ setting $a \circ a=c_{0}^{a}, a \circ c_{k}^{a}=c_{k}^{a} \circ a=c_{k+1}^{a}$ for $k=0,1,2, \ldots$, and $a \circ b=b \circ a=g^{\mathbf{A}}(a, b)$ otherwise. This operation $\circ$ is commutative, and is also total by $(q 2)$.

Let $\mathbf{B}$ be an algebra consisting of all elements of $\mathbf{A}$ and operations o, $h^{\mathbf{A}}$, $u^{\mathbf{A}}$ only. Then a set $C \subseteq A$ is closed under operations in $\mathbf{B}$ if and only if $C$ is closed under operations in $\mathbf{A}$. Hence, $\mathbf{B}$ has the same distributive subalgebra lattice as $\mathbf{A}$, is generated by $x, y$ and is not cyclic.

If $(a, b) \in \mathcal{X}$, then $b=c_{k}^{a}$ for some $k$, so $a \circ c_{k-1}^{a}=c_{k-1}^{a} \circ a=b$. if $(a, b) \in \mathcal{Y}$, then there are $k=0,1,2, \ldots$ and $d \in A$ such that $f_{k}^{\mathbf{A}}(d)=a$, $f_{k+1}^{\mathbf{A}}(d)=b$. Hence, $a=c_{k}^{d}, b=c_{k+1}^{d}$ and $d \circ c_{k}^{d}=c_{k}^{d} \circ d=b$. Thus by $(q 3)$, equations $a \circ z=z \circ a=b$ have solutions for each $a, b$.

We show that these equations have exactly one solution. Let $a \circ u=b=$ $u \circ a$ and $a \circ w=b=w \circ a$. If $(a, u),(a, w) \in \operatorname{dom}\left(g^{\mathbf{A}}\right)$, then $u=w$ because $g^{\mathbf{A}}$ has PDP. Assume $(a, u) \notin \operatorname{dom}\left(g^{\mathbf{A}}\right)$. Then $a=u$ or $(a, u) \in \mathcal{X}$ or $(u, a) \in \mathcal{X}$. If $a=u$ or $(a, u) \in \mathcal{X}$, then $u=c_{l}^{a}$ for some $l=-1,0,1, \ldots$ and $b=a \circ u=c_{l+1}^{a}$. So $(a, b) \in \mathcal{X}$ and by $(q 3),(a, w) \notin \operatorname{dom}\left(g^{\mathbf{A}}\right)$. If $a=w$ or $(a, w) \in \mathcal{X}$, then $w=c_{j}^{a}$ for some $j=-1,0,1, \ldots$, hence $c_{l+1}^{a}=b=a \circ w=c_{j+1}^{a}$ which implies $j=l$, so $w=u$. If $(w, a) \in \mathcal{X}$, then $a=c_{l}^{w}$ and $b=c_{l}^{w} \circ w=c_{l+1}^{w}$, so $(a, b) \in \mathcal{Y}$, a contradiction with $(q 5)$. Now by symmetry we can assume $(u, a),(w, a) \in \mathcal{X}$. Then $a=c_{l}^{u}=c_{j}^{w}$ and $b=c_{l+1}^{u}=c_{j+1}^{w}$, so $(a, b) \in \mathcal{Y}$ and by $(q 4), w=u$.

Summarizing, the carrier of $\mathbf{B}$ with $\circ$ is a commutative (total) quasigroup $\mathcal{Q}$. Let $\backslash$ be the division corresponding to $\circ$. Then $h^{\mathbf{A}} \subseteq \backslash$ and $u^{\mathbf{A}} \subseteq \backslash$. Hence, if a set is closed under $\circ$ and $\backslash$, then it is closed under all operations of $\mathbf{B}$. Conversely, take a subalgebra $\mathbf{C}$ of $\mathbf{B}$ and $a, b \in C$. If $(a, b) \in \operatorname{dom}\left(h^{\mathbf{B}}\right)$ or $(a, b) \in \operatorname{dom}\left(u^{\mathbf{B}}\right)$, then $a \backslash b$ also belongs to $C$. Thus assume $(a, b) \notin \operatorname{dom}\left(h^{\mathbf{B}}\right)$ and $(a, b) \notin \operatorname{dom}\left(u^{\mathbf{A}}\right)=\mathcal{Y}$. By $(q 3),(a, b) \in \mathcal{Y} \cup \mathcal{X}$, so $(a, b) \in \mathcal{X}$. Then $b$ and $a \backslash b$ belong to the subalgebra generated by $a$. Hence, $\mathcal{Q}$ has a distributive subquasigroup lattice (the same as $\mathbf{B}$ ), is generated by two elements and not cyclic.

Open Access. This article is distributed under the terms of the Creative Commons Attribution Noncommercial License which permits any noncommercial use, distribution, and reproduction in any medium, provided the original author(s) and source are credited.

\section{References}

[1] Bartol, W., Rosselló, F., Rudak, L.: In: Rosselló, F. (ed.) Lectures on algebras, equations and partiality. Technical report B-006, Univ. Illes Balears, Dept. Ciencies Mat. Inf. (1992)

[2] Jónsson, B.: Topics in Universal Algebra. Lecture Notes in Mathemathics 250. Springer, New York (1972) 
[3] Pióro, K.: On finite quasigroups whose subquasigroup lattices are distributive. Quasigroups Relat. Syst. 15, 309-316 (2007)

[4] Schmidt, R.: Subgroup Lattices of Groups. Walter de Gruyter, New York (1994)

Konrad Pióro

Institute of Mathematics

University of Warsaw

ul. Banacha 2

02-097 Warsaw

Poland

e-mail: kpioro@mimuw.edu.pl

Received: June 23, 2009.

Revised: October 29, 2009.

Accepted: December 15, 2009. 